\title{
Implementasyon ng online learning sa Leyte Normal University: Saloobin ng mga mag-aaral
}

Dadacay, Alyssa $\$
Leyte Normal University, Philippines (alyssadadacay1020@gmail.com)

Guba, Arjelyn

Leyte Normal University, Philippines (gubaarajelyn@gmail.com)

Costiniano, Angel

Leyte Normal University, Philippines ( $\underline{\text { costinianoangel@gmail.com) }}$

Gumba, Kimberly

Leyte Normal University, Philippines (kimberlygumba@gmail.com)

Lendio, Jenecyl

Leyte Normal University, Philippines (jenecyllendio5@gmail.com)

Lopez, Christian Dale

Leyte Normal University, Philippines (ainghalos@gmail.com)

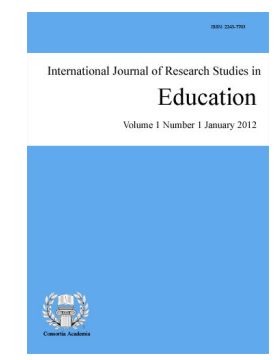

ISSN: 2243-7703 Online ISSN: 2243-7711

OPEN ACCESS

\section{Abstract}

This research is about students' perception regarding the implementation of online learning. This research was conducted to find out the different perceptions regarding the implementation of online learning focused on online learning materials, strategies and support. The researcher used a questionnaire or survey questionnaire as a primary instrument in collecting data where the respondents are the first-year students at Leyte Normal University in the academic year 2020-2021 with a total number of one thousand five hundred and seventy (1570). The total number of respondents was determined using the table by Krejcie and Morgan (1970). Meanwhile, in determining the respondents the Quota Sampling technique was used and the research design was cross-sectional survey design. The researcher found that the students agreed with the use of online learning materials because they are accessible such as power point and others. It also emerged from this research that there was uncertainty as to the student's perceptions towards the asynchronous classes but majority of the items were agreed. In the items for online learning support, most of the students agreed. A good intervention in this research is to expand the use of online learning, strategies and support to develop deeper learning and meet each other's needs in online learning.

Keywords: implementation, perception, online learning materials, online learning strategies, online learning support 


\section{Implementasyon ng online learning sa Leyte Normal University: Saloobin ng mga mag-aaral}

\section{Introduksiyon}

Sa kasalukuyang sistema ng edukasyon dulot ng Covid-19 ay hindi na ginagawang harapan ang klase sa loob ng paaralan kundi sa pamamagitan na lamang ng mga teknolohiya, internet at mga digital platforms. Alinsunod sa Republic Act (RA) No.7722 o mas kilala bilang "Higher Education Act 1994" o "Bayanihan To Heal As One Act" at ayon sa bisa ng Commission en Banc (CEB) ay nagpahayag ng mga sumusunod na alituntunin at ipatupad ang flexible learning sa pampubliko at pampribadong institusyon (Bilbao et al., 2014).

Ayon kay Bernard et al. (2014) ang online learning ay isang paraan na nakapaloob sa tinatawag na blended learning at saklaw ang pag-aaral sa klasrum na birtual, tulad ng Zoom, Google Classroom, Edmodo, Schoologoy at iba pa. Batay sa mga pag-aaral na isinagawa ni Burke (Burke, 2020; del Castillo, 2021), ang edukasyon sa panahong ito ng pandemya ay magiging mapanghamon lalo na ang online learning. Sinasabi sa kanyang artikulo na sa pagkatuto ng online ay magiging hamon hindi lamang ang mga kagamitan at internet connection, kundi maging ang emosyonal at saykolohikal na aspeto.

Sa pag-aaral ni Jaggars (2020), sinasabi ng mga estudyante na hindi epektibo ang online class dahil mahirap matuto at nagiging limitado ang interaksiyon ng guro at estudyante. Sa kabilang banda isinaad sa pag-aaral nina Laili at Nashir (2021), na parehong may positibo at negatibong saloobin ang mga mag-aaral hinggil sa online learning, ngunit higit na binigyang-diin na dapat ay magkaroon ng sapat na mga materyales, suporta at estratehiya online para sa mas maayos na online learning na karanasan para sa mga mag-aaral.

Maraming kabataan ang nahihirapan sa bagong sistema ng edukasyon ngayon dahil ang pagtuturo ay maaaring synchronous o asynchronous. Sa una, ang guro at mga mag-aaral ay may interaksiyong real time o sabayan sa aktuwal o itinakdang oras ng klase na naging dagok para sa mga estudyante na makasabay at makapasok sa birtuwal na klase dahil sa maraming suliranin. Sa ikalawa naman, maaaring wala ng interkasiyon ang guro at mga mag-aaral kung saan natututo ang mag-aaral sa pamamagitan ng iba't ibang kagamitang pampagkatuto gaya ng mga video presentation/clips, modyuls, ebooks at iba pa. Maaari rin gumamit ng aplikasyon ang estudyante gaya ng Zoom, Skype, Google Hangouts, Facebook, Messenger upang kumonsulta sa guro.

Sa artikulo ng Manila Times na pinamagatang Learning in the new normal - through the eyes of Filipino students, naibahagi ng ilang mag-aaral ang maraming pagsubok at suliranin sa pag-aaral sa panahon ng pandemya. Unang-una nariyan ang internet connection (Abu, 2020). Batay sa Speedtest Global Index, ang Pilipinas ay pang-109 sa 139 na bansa pagdating sa mobile internet speed na nangangahulugang, ang pagsasagawa ng online classes ay lubos na apektado. Nariyan ang hindi pag-play ng video presentation, hindi makapasok sa link, nauubos ang data at kapag matagal nang nakababad ang mga estudyante sa screen, umiiksi ang atensyon span nila (Devio, 2021).

Sa pananaliksik na ito ay binigyang-pansin ang mga saloobin at mahahalagang aspeto na hindi natukoy sa mga naunang pananaliksik, pag-aaralan ang iba't ibang saloobin tungkol sa implementasyon ng online learning na nakatuon sa online learning materials, online learning strategies at online learning support. Ang pananaliksik ay may pagkakatulad sa mga naunang pananaliksik ngunit naiiba sa lawak ng saklaw at layunin ng pananaliksik. Mula 1980, ang teknolohiya ay radikal na binabago ang pang-araw-araw na buhay, komunikasyon at edukasyon. Ang pananaliksik na ito ay mahalaga sa kasulukuyang sistema ng pagaaral, at sa susunod pang mga panahon lubos na magagamit ang pananaliksik na ito upang matugunan ang mga problema o saloobin batay sa implementasyon ng online learning. 


\subsection{Kaligirang Teoretikal}

Sa pag-aaral na ito, ginamit ang unang teorya kung saan nakasalig ang konsepto ng teoryang Online learning Through Technology nina Redmond M. De Vera, Suzanne Ann Rosales at Jeremiah Villanueva ay tungkol sa konsepto na binubuo sa interaksiyon ng mga mag-aaral pagdating sa pamamaraan ng makabagong edukasyon sa ngayon (Hiltz et al. 2005). Dagdag pa rito, binigyang-pansin kung paano nakaapekto ang paggamit ng teknolohiya sa mga mag-aaral sa kanilang pang-araw-araw. Sa pamamagitan ng teoryang ito ay malalaman ang mga online learning materials na maaaring gamitin ng guro at estudyante, mga estratehiya at suporta ng guro sa mga mag-aaral. Lubos na malaking ang kontribusyon at ambag ng teknolohiya sa buhay ng bawat indibiduwal lalo na sa makabagong sistema ng edukasyon ngayon. Malaki ang ginampanan ng teknolohiya sa panahon ng pandemya dahil natutugunan nito ang mga pangangailangan at kaalaman ng isang mag-aaral.

Kaugnay nito ay sanligan din ang ikalawang teorya Connectivism: A learning theory for digital age ni Goerge Siemens, nakatuon sa mga mag-aaral kung saan natuto kaakibat ng kaniyang kapaligiran, halimbawa ay mga database, organisasyon o mga makabagong teknolohiya. Gamit ang teoryang ito ay mababatid ang kahalagahan at gamit ng online learning sa panahon ng pandemya na ginagamitan ng laptop, computer, cellphone, tablet at iba pa. Sa pamamagitan ng mga kagamitang ito ay nabibigyang pagkakataon na makipag-uganayan at manatiling nakakaugnay ang guro at mag-aaral sa iba't ibang lugar sa pamamagitan ng internet. Sa connectivism, ang teknolohiya ay nagkakaroon ng parte sa external learning process ng mga estudyante (Siemens, 2005).

Pinagtibay din sa ikatlong teoryang Contructivism Learning Theory, na nagkakaroon ng pagkatuto ang isang tao, at nakakayang gumawa ng makabuluhan batay sa kanyang karanasan. Sa pananaw na ito, nabibigyan ng bagong pagkatuto ang mag-aaral gamit ang mga tanong ng guro at kanyang malikhaing paraan. Nakita nito ang kahalagahan ng paglinang ng kognitibong aspeto ng mag-aaral sa pamamagitan ng pagbibigay ng ebidensya mula sa alternatibong pagtuturo na ginagamitan ng siyensya at ng iba't ibang pangteknolohiyang kagamitan. Sa pamamagitan ng teoryang ito, mabibigyang pansin ang mga suporta online ng mga mag-aaral sa online learning (Kneller, 2009).

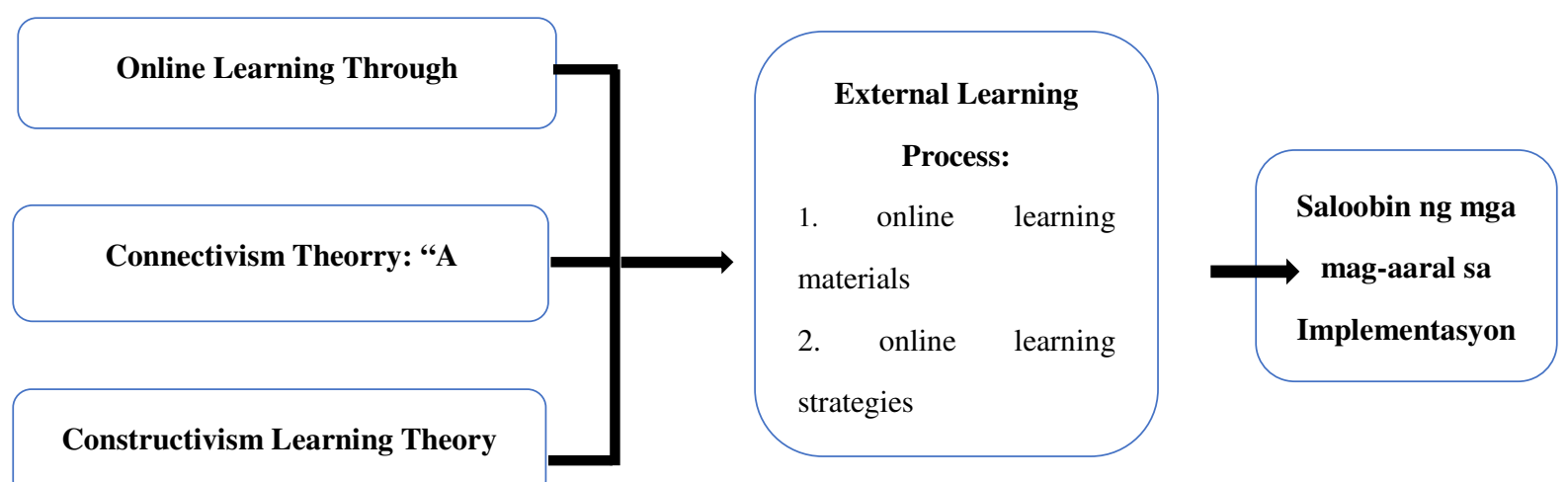

Figyur 1: Balangkas ng Pag-aaral

Sa Figyur 1 makikita ang balangkas ng pag-aaral kung saan sa unang tatlong (3) kahon ay ang mga teoryang ginamit: Online Learning Through Technology, Connectivism Theory: "A learning Theory for Digital Age" at Constructivism Learning Theory. Sa gitnang bahagi naman ay mga baryabol ng pag-aaral: online learning materials, strategies at support at sa huling kahon ay resulta ng pananaliksik kung saan ito ang saloobin ng mga mag-aaral sa implementasyon ng online learning

\subsection{Paglalahad ng Suliranin}

Layunin ng pananaliksik na ito na malaman ang iba't ibang saloobin ng mga mag-aaral hingil sa 
implementasyon ng online learning sa akademikong taon 2020-2021. Tiniyak ng pananaliksik na: (1) Matukoy ang saloobin ng mga mag-aaral hinggil sa implemenstasyon ng online learning partikular sa: (a) online learning materials; (b) online learning strategies; at (c) online learning support; at (2) Matukoy kung mayroon bang mahalagang kaugnayan sa isa't isa ang mga salik na online learning materials, online learning strategies at online learning support.

\section{METODOLOHIYA}

Ang pananaliksik na ito ay isang kwantitatibong pag-aaral at ginamit ang disenyong cross-sectional sa pananaliksik upang malaman ang kasalukuyang saloobin ng mga mag-aaral sa implementasyon ng online learning. Ito ay sumusukat sa populasyon ng mga mag-aaral na natuto sa online learning gamit ang iba't ibang kagamitan gaya ng google meet, zoom at marami pang iba na ginagamit ng mga guro bilang alternatibong paraan tungo sa pagkatuto ng bawat mag-aaral ngayong panahon ng pandemya.

Nasa Quota Sampling teknik naman ang ginamit sa pagtukoy ng mga respondente upang maging pantay ang ginawang pag-aaral sa mga mag-aaral na nasa unang taon ng Leyte Normal University, Tacloban. Ginamit ng mga mananaliksik ang talahanayan nina Krejcie at Morgan (1970) sa pagkuha ng sample size na may kabuuang bilang na tatlongdaan at sampu (310). Ang mananaliksik ay gumamit ng talatanungan o survey questionnaire bilang pangunahing instrumento sa pagkalap ng datos na magagamit sa pag-aaral. Ginamit ng mananaliksik ang Likert Scale ni Likert (1932) na ang karaniwang pinagpipilian ay lima hanggang pito na nakaayos na pagpipilian. Ang talatanungan na ito ay mayroong isang bahagi tungkol sa saloobin ng mga mag-aaral sa implementasyon ng online learning na binubuo ng sumusunod. Sa bahaging ito rin ay pinagbatayan sa pagkuha ng impormante ay ang mga sumusunod na external learning process ng mga mag-aaral. Una (1) ay ang online learning materials tulad ng modyuls, video presentation/clips, links, ebook atbp. na mga kagamitan sa pagkatuto na kadalasang ginagamit sa online learning. Pangalawa (2) ay ang online learning strategies na tumutukoy ito sa mga estratehiya ng mga guro sa pamamagitan ng online learning kagaya ng pagkakaroon ng synchronous at asynchronous na klase sa googe meet, zoom atbp. at ang pangatlo (3) ay ang online learning support na tumutukoy sa mga suporta na binibigay ng mga guro sa kaniyang mag-aaral online. Ang mga tanong o ang nilalaman ng sarbey kwestyuner na ginamit sa pananaliksik na ito ay ayon sa ginamit ng naunang pag-aaral nina Khan at Jumani (2010) at Fitri (2020). Gumamit ang mananaliksik ng Likert Scale at sa kompyutasyon ng bahagdan ay ginamit ang Weighted Mean na pamamaraan.

\section{Mga resulta at diskusyon}

Sa bahaging ito ay makikita ang pagsusuri at pagpapakahulugan ng mga datos batay sa isinagawang pangangalap tungkol sa saloobin ng mga mag-aaral sa implementasyon ng online learning sa Leyte Normal University. Nahahati sa apat (4) na bahagi ang talahanayan para sa paglalahad at pagpapakahulugan ng mga datos.

Ang Talahanayan 1 ay nagpapakita sa mga aytem na nagsasaad ng mga saloobin ng mga respondente tungkol sa online learning materials. Anim na aytem (6) ang nagsasaad ng sumasang-ayon, tatlong aytem (3) ang nagsasaad nang walang katiyakan at may isang (1) aytem na kung saan lubos na sumasang-ayon ang mga respondente. Makikita na ang aytem na "Mas madaling sagutan ang mga materyales sa online learning." at "Madaling gumamit ng mga projector at video screen para sa paglalahad ng reports." ang dalawang may mababang mean na nagsaad na walang katiyakan na sagot. Ang aytem naman na "Gumagamit ang guro ng mga presentation software tulad ng Powepoint Presentation atbp." ang may pinakamalaking mean na siyang lubos na sinasang-ayonan. Ang naging kabuuang mean ng salik na online learning materials ay 3.61 na nagsasaad na sumasang-ayon ang mga respondente sa mga aytem na nakasaad dito.

Ang resultang ito ay maihahalintulad at maiuugnay sa natalakay sa literatura ng pag-aaral tungkol sa artikulo ni Dela Cruz (2020). Itinampok sa artikulong ito na mapanghamon ang online learning hindi lamang 
dahil sa mga salik sa suporta o materyales kundi dahil na rin sa mahirap ang magpokus sa birtuwal na klase dahil sa mga distraksiyon sa kanya-kanyang kabahayan. Sinang-ayonan rin ito ng pag-aaral nina Agung et al. (2020) na batay sa saloobin ng mga mag-aaral ay malaki talaga ang kanilang suliraning kinakaharap sa mga materyales at kagamitan sa online learning. Bagamat pinagsisikapan ng mga guro at mag-aaral ang maghanda at magkaroon ng mga sapat na Online Learning Materials ay may mga pagkakataong hindi pa rin ito nakasasapat.

\section{Talahanayan 1}

Online Learning Materials

\begin{tabular}{|c|c|c|}
\hline Mga Pahayag & Mean & Interpretasyon \\
\hline $\begin{array}{l}\text { Madaling ma-access ang mga kagamitang pampagkatuto gaya ng modyuls, video } \\
\text { presentation/clips, links, ebooks atbp. }\end{array}$ & 3.72 & Sumasang-ayon \\
\hline $\begin{array}{l}\text { Gumagamit ng wastong mga bidyo, larawan at audio bilang pandagdag na suporta } \\
\text { sa. pagkatuto online }\end{array}$ & 4.03 & Sumasang-ayon \\
\hline $\begin{array}{l}\text { Malawak at maraming impormasyon ang nakukuha sa kagamitan o materyales sa } \\
\text { online learning. }\end{array}$ & 3.59 & Sumasang-ayon \\
\hline Mahirap maunawaan ng mga estudyante ang mga materyales sa pagkatuto online. & 3.56 & Sumasang-ayon \\
\hline $\begin{array}{l}\text { Hindi nababagot ang mga estudyante kapag letra, larawan at iilan pang bagay ang } \\
\text { nakikita sa screen sa online na klase. }\end{array}$ & 3.20 & Walang katiyakan \\
\hline Madaling gumamit ng mga projector at video screen para sa paglalahad ng reports. & 3.05 & Walang katiyakan \\
\hline Mas madaling sagutan ang mga materyales sa online learning. & 3.03 & Walang katiyakan \\
\hline $\begin{array}{l}\text { Epektibo ang pagkatuto sa pag-access sa mga kagamitan o materyales sa google } \\
\text { form, google drive atbp. }\end{array}$ & 3.42 & Sumasang-ayon \\
\hline \multirow{3}{*}{$\begin{array}{l}\text { Mahirap ang magpokus sa mga aralin sa birtuwal na klase. } \\
\text { Gumagamit ang guro ng mga presentation software tulad ng Powepoint } \\
\text { Presentation atbp. }\end{array}$} & 4.11 & Sumasang-ayon \\
\hline & 4.39 & Lubos na Sumasang-ayon \\
\hline & 3.61 & Sumasang-ayon \\
\hline
\end{tabular}

\section{Talahanayan 2}

Online Learning Strategies

\begin{tabular}{|c|c|c|}
\hline Mga Pahayag & Mean & Interpretasyon \\
\hline $\begin{array}{l}\text { 1. Mayroong pinagkakasunduang oras at araw para sa synchronous at } \\
\text { asynchronous na klase. }\end{array}$ & 4.14 & Sumasang-ayon \\
\hline 2. Ang guro at mag-aaral ay may interaksiyon sa itinakdang oras na klase. & 4.00 & Sumasang-ayon \\
\hline 3. Ang guro ay nagtatanong at bukas sa talakayan. & 4.16 & Sumasang-ayon \\
\hline $\begin{array}{l}\text { 4. Laging ipinapaliwanag ang gawain sa pag-aaral sa online bago magbigay ng } \\
\text { takdang aralin. }\end{array}$ & 3.54 & Sumasang-ayon \\
\hline 5. Sapat ang pagtatalakay sa bawat paksa at mga gawain sa asynchronous na klase. & 3.35 & Walang katiyakan \\
\hline 6. Palaging ginagawang kawili-wili aat nauugnay ang birtuwal na klase ng guro. & 3.55 & Sumasang-ayon \\
\hline 7. Nagbibigay ang guro ng iba't ibang mga paran upang masukat kung ano ang & 4.04 & \\
\hline natutunan ng estudyante (test, projects, discussion atbp.) & & Sumasang-ayon \\
\hline 8. Interaktibo ang mga gawaing pangkatan sa online learning. & 3.33 & Walang katiyakan \\
\hline 9. Mabisang ginagamit ng guro ang oras ng klase online. & 3.81 & Sumasang-ayon \\
\hline $\begin{array}{l}\text { 10. Ipinipresenta ang mga materyales sa iba't ibang paraan (hands-on, group } \\
\text { written, orally atbp.) }\end{array}$ & 3.99 & Sumasang-ayon \\
\hline Kabuuan & 3.79 & Sumasang-ayon \\
\hline
\end{tabular}

Talahanayan 2 ay nagpapakita sa mga aytem ukol sa online learning strategies. Ang kabuuang reaksiyon ng mga respondente ay may mean na 3.79 na ibig sabihin sumasang-ayon. Ang dalawang aytem na "Ang guro ay nagtatanong at bukas sa talakayan." at "Mayroong pinagkakasunduang oras at araw para sa synchronous at asynchronous na klase." ay nabibigyan nang pinakamalaking pagsang-ayon ng mga respondente na umabot sa 4.16 ant 4.14. May dalawang aytem naman na kung saan ay walang katiyakan ang naging sagot nang mga respondente. Ito ay ang "Palaging ginagawang kawili-wili at nauugnay ang birtuwal na klase ng guro." at "Interaktibo ang mga gawaing pangkatan sa online learning." na nabigyan lang nang mean na 3.33 at 3.35.

Maiuugnay ang resultang ito sa pag-aaral ni Dwidienawati et al. (2020) na pinamagatang Digital Communication in Online Learning during Mobility Restriction na bagamat nagugustuhan ng mga mag-aaral ang online learning, ilan sa mga suliranin nila ay ang internet connection, hindi masyadong naiintindihan ang mga aralin at kakulangan ng interaksyon sa parehong kaklase at guro. Ganoon din ito ay sinasang-ayunan ng 
pag-aaral nina Mallilin et al. (2020) na nagsaad na " Results of the study show an establish proper schedule of students in their regular classes during the period of Covid 19 pandemic and develop proper strategy in teaching during their lecture hours and provide focus to support students in their learning process and undertake initiatives despite of the limited resources in online teaching, identify the means of delivery mode of teaching based on the needs of students in their learning process, support students in their new modality of instruction in their online classes that boost their learning potential ability, and school provides guidelines for the online learning of student tools during Covid 19 pandemic to ensure better education." Sapagkat nakikita sa resulta na bagamat mataas ang pagsang-ayon sa ibang aytem nariyan pa rin ang mga suliranin at mga bagay na kailangan pang paunlarin o ayusin.

\section{Talahanayan 3}

Online Learning Support

\begin{tabular}{|c|c|c|}
\hline Mga Pahayag & Mean & Interpretasyon \\
\hline 1. Nagbibigay ng fidbak ang guro sa lahat ng mga gawain sa online na klase. & 3.47 & Sumasang-ayon \\
\hline $\begin{array}{l}\text { 2. Pinapadali at sinusubaybayan ng guro ang naaangkop na pakikipag-ugnayan sa } \\
\text { mga mag-aaral. }\end{array}$ & 3.59 & Sumasang-ayon \\
\hline $\begin{array}{l}\text { 3. Ang guro ay may kakayahang umangkop sa pagharap sa mga pangangailangan } \\
\text { ng mga estudyante (due dates, absences at make-up exams). }\end{array}$ & 3.93 & Sumasang-ayon \\
\hline $\begin{array}{l}\text { 4. Komukonsulta ang guro sa estuyante gamit ang aplikasyon tulad ng zoom, } \\
\text { facebook, messenger at iba pang digital plaforms. }\end{array}$ & 4.15 & Sumasang-ayon \\
\hline $\begin{array}{l}\text { 5. Hindi pwedeng ipadala ang mga babasahin at lekyur na nakasulat sa } \\
\text { pamamagitan ng pagtawag sa telepono, cellphone at text. }\end{array}$ & 3.68 & Sumasang-ayon \\
\hline $\begin{array}{l}\text { 6. Mahinahon at magalang ang guro sa pagbbibigay ng suhestyon at panuto sa } \\
\text { estudyante. }\end{array}$ & 4.06 & Sumasang-ayon \\
\hline $\begin{array}{l}\text { 7. Ang guro ay nagbibigay ng lingguhang mga takdang-aralin sa halip na } \\
\text { pang-araw-araw na mga deadline. }\end{array}$ & 4.14 & Sumasang-ayon \\
\hline 8. Mas nakapagbibigay ng motibasyon ang online learning. & 2.35 & Hindi sumasang-ayon \\
\hline $\begin{array}{l}\text { 9. Gumagamit ang guro ng email upang ipadala ang mga babasahing nakarekord } \\
\text { atbp. sa estudyante. }\end{array}$ & 4.13 & Sumasang-ayon \\
\hline 10. Madaling magkaroong ng "Question and Answer" na bahagi sa online class. & 3.29 & Walang katiyakan \\
\hline Kabuaan & 3.68 & Sumasang-ayon \\
\hline
\end{tabular}

Talahanayan 3 ay nagsasaad sa mga aytem sa online learning support. Makikita na ang aytem na "Mas nakapagbibigay ng motibasyon ang online learning." ay may mean na hindi sinasang-ayunan ng mga respondente. Walang katiyakan naman ang naging mean na sagot ng mga respondente ukol sa madaling pagkakaroon ng "Question and Answer" na bahagi sa online class. Ang dalawang aytem ang nabigyan ng maliit na mean kaya makikita na ang kabuuang mean ay nasa 3.68 o sumasang-ayon ang mga respondente. Makikita rin ang unang tatlong aytem na may mataas na mean ay sinasang-ayunan ng mga respondente ay nasa $4.15,4.14$ at 4.13 .

Ang pagtalakay at resulta sa talahanayan 3 ay sinasang-ayunan ng pag-aaral ng OECD (2020) tungkol sa pagpapatibay sa online learning. Inilahad nila na napakalaki ng ginagampanan ng Online Learning Support sa pagkatuto ng mga mag-aaral kung kaya marapat na makapagbigay ng isang aktibo at makahulugang pagsuporta ang parehong mga guro at magulang sa mga mag-aaral. Natalakay din sa pag-aaral nina Subekti at Wacana (2020) ang kakulangan ng online learning support bilang isa sa mga suliranin sa pagkatuto sa panahon ng pandemya, naisaad sa kanilang pag-aaral na bagamat ang online learning ay isinagawa upang patuloy pa rin ang pagkatuto at edukasyon, dapat ay magkaroon ng mga hakbangin upang mapaunlad pa ang sistema at mas mabigyan pa ng sapat na kaalaman at kakayahan ang mga guro para maging mas mabisa ang online learning at pagbibigay ng online learning support.

Talahanayan 4 ay nagpapakita ng ugnayan ng mga salik sa online learning. Makikita sa resulta gamit ang korelasyon ayon kay Spearman na may mahalagang pagkakaugnay ang tatlong aspeto sa online learning. Basi sa mga resulta ng p-value, ito ay nasa 0.000 na ang ibig sabihin ay "significant" ang nakikitang korelasyon o pagkakaugnay nang tatlo. Ang ibig sabihin nito na ang mga respondente ay nagkakaisa sa kanilang mga kasagutan at ito ay may malakas na pag kakapareho at walang gaanong pagkakaiba. Nagpapakita ang resulta na 
ang datos na nakuha ay may sapat na dami upang sabihin na pwedeng gawan konklusyon ang pag-aaral na ito. Masasabing ang saloobin ng mga mag-aaral ay maaring makuha at mabigyan pagpapakahulugan batay sa tatlong salik na mayroon ang kasalukuyang pag-aaral. Ito ay sinusuportahan at maiiuugnay sa isinagawang pag-aaral nina Laili at Nashir (2021) na parehong may positibo at negatibong saloobin ang mga mag-aaral hinggil sa online learning, ngunit higit na binigyang diin na dapat ay magkaroon ng sapat na mga materyales, suporta at estratehiya online para sa mas maayos na online learning na karanasan para sa mga mag-aaral.

\section{Talahanayan 4}

Mahalagang Kaugnayan ng mga Salik Online Learning

\begin{tabular}{lll}
\hline \multicolumn{1}{c}{ Salik } & P-value & \multicolumn{1}{c}{ Interpretasyon } \\
\hline Onine Learning Material & 0.000 & Pareho ang pananaw o Sumasang-ayon sa iba \\
Onine Learning Strategies & 0.000 & Pareho ang pananaw o Sumasang-ayon sa iba \\
Onine Learning Support & 0.000 & Pareho ang pananaw o Sumasang-ayon sa iba \\
\hline
\end{tabular}

\section{Konklusyon at rekomendasyon}

\subsection{Konklusyon}

Batay sa mga natuklasan ng mga mananaliksik mula sa resulta ng mga datos na nakalap mula sa mga respondent napatunayan sa pag-aaral na ito ang sumusunod:

> Mula sa kabuuang resulta sa sampung aytem na naglalaman ng mga saloobin ng mga mag-aaral tungkol sa implementasyon ng online learning partikular na sa paggamit ng online learning materials, ang mga mag-aaral ay sumasang-ayon sa mga aytem na ito. Na kung saan makikita na malaki ang bilang na nagsasabing madaling maka-access sa mga kagamitang pampagkatuto, gumamit ng wastong kagamitan bilang pandagdag suporta sa pagkatuto, maraming impormasyon ang makukuha sa online, at ang paggamit ng mga materyales kagaya na lamang ng powerpoint, google form at google drive. Pabor din ang mga mag-aaral na mahirap na magpukos sa mga birtuwal na klase.

$>\quad$ Walang katiyakan ang naging saloobin ng mga mag-aaral patungkol sa kasapatan ng asynchronous na klase sa pagtalakay sa bawat paksa at gawain gayundin sa pagkaroon ng interaktibong mga gawaing pangkatan. Subalit ang kabuuang reaksiyon ng mga respondente ay may mean na 3.79 na ibig sabihin sumasang-ayon sa mga aytem ang mga mag-aaral, kaya naman ang saloobin ng mga mag-aaral ay pabor sa mga ginamit na mga online learning strategies ng mga guro sa pagtuturo online.

$>\quad$ Para naman sa mga saloobin ng mga mag-aaral sa implementasyon ng online learning partikular na sa pagbibigay online learning support, mula sa sampung aytem mayroong isang pahayag na hindi sinang-ayonan ng mag-aaral, na nagsasabing mas nakapagbibigay motibasyon ang online learning. May isa rin aytem kung saan ayon sa mga mag-aaral ay hindi nila matiyak o walang katiyakan ang kanilang tugon tungkol sa madaling magkaroon ng "Question and Answer" na bahagi sa online class. Ngunit sa kabuuan nito makikita ang unang tatlong aytem na may mataas na mean na sinasang-ayunan ng mga mag-aaral ay nasa 4.15, 4.14, 4.13 na nagsasabi na nagkaroon ng online learning support, kagaya na lamang ng pagkaroon ng fidbak, pagsubaybay ng mga guro sa mga mag-aaral, pag-angkop ng mga guro sa mga pangangailangan ng mga studyante, pagkaroon ng konsultasyon, pagiging mahinahon at magalang ng guro, pagsaalang-alang sa pagbibigay deadline, at paggamit ng email para sa dagdag na materyales lalong-lalo na sa mga mag-aaral na hindi nakadalo.

\subsection{Rekomendasyon}

Batay sa mga naging kongklusyon ng pag-aaral, inirekomenda ng mga mananaliksik ang sumusunod:

Para sa CHED, kailangan nilang magbigay ng sapat na mga kagamitang pampagtuturo, mga training at 
seminar) na makatutulong na paunlarin ang mga kaalaman at kakayahan ng mga guro sa makabagong sistema ng edukasyon (online learning) ng sa gayon ay makabuo ang mga guro ng wastong online learning strategies at makapagbigay ng akmang online learning support sa bawat mag-aaral. Palawakin pa at patuloy na gamitin ang mga online learning materials na nakasanayan na ng mga mag-aaral nang sa ganoon ay lalo pa nilang mahasa ang kanilang kaalaman at mapaunlad ang kakayahan sa paggamit ng mga materyales online. Ang paggamit ng powerpoint, google form at google drive ay palawakin pa upang lumawig at lumawak ang sakop ng mga online learning materials na nagagamit at maaari pang magamit sa online class. Sa mga nahihirapan na magbigay ng pokus sa birtuwal na klase, maghanap ng maayos, maganda at tahimik na lugar o sa inyong tahanan na walang ingay o makapagbibigay distraksiyon. Magkaroon din ng bukas at maayos na komunikasyon sa guro at pamilya upang matugunan at matulungan, nang sa gayon magkaroon ng maayos na espasyo at lugar na kung saan mas makapagbibigay ng pokus sa online class.

$>$ Para sa mga guro, patuloy na sikapin ng mga ang pakikiisa sa mga gawain para sa personal at professional development ng sa gayon, ay lumawak pa ang kaalaman at kakayahan upang makagamit pa ng iba't ibang estratehiya at makapagbigay suporta sa mga mag-aaral at maging makabuluhan at interaktibo pa rin ang bawat online learning session. Patuloy na palawigin at paunlarin ang mga online learning strategies na ginagamit sa online class dahil higit na nakatutulong ito sa proseso ng pagkatuto. Sikapin na makadiskubre ng iba pang pamamaraan upang gawing mas interaktibo at pukawin ang interes ng mga mag-aaral sa mga isinasagawang asynchronous classes. Bigyang-pansin ang oras ng mga pinagkakasunduang asynchronous classes upang masigurado na ang bawat meeting o klase ay nakasasapat upang masigurado ang malawak na pagkatuto at malalim na pag-unawa sa mga araling tinalakay. Ipagpatuloy ang mga isinasagawang online learning support dahil malaki ang naitutulong nito upang matuto at walang mag-aaral ang nahuhuli sa klase sa gitna ng iba't ibang suliranin sa online class na kinakaharap ng mga mag-aaral sa gitna ng pandemya. Bigyang pokus ang pagkakaroon ng "Question and Answer" upang makita at masukat ang kaalaman at pag-unawa ng mga mag-aaral sa paksang tinatalakay. Bigyang-tuon din ang pagkakaroon ng mabuti at bukas na komunikasyon nang sa gayon ay batid ng guro at ng iba pang may kakayahan kung papaano makapagbibigay ng suporta online.

> Para sa mga mag-aaral ay kinakailangan na bigyan ng sapat na kaalaman patungkol sa paggamit ng mga online learning materials at mabigyan ng sapat na kagamitan upang makasabay sa bagong sistema ng edukasyon at pagkatuto sa kasalukuyan. Marapat din maging bukas lagi ang mag-aaral sa komunikasyon sa kanilang guro upang magkaroon ng maayos na pagkakaunawaan at pagkakaisa sa mga kasunduan hinggil sa mga gawain at malaman kung paano at anong mga online learning strategies at support ang kanilang kailangan.

> Para sa mga magulang, kailangan na mabigyan din ng sapat na kaalaman ang bawat mga magulang sa mga pagbabago ng edukasyon sa panahon ngayon dahil sila ang magsisilbing mga guro sa kanilang mga anak. Kaya nararapat na sila'y magkaroon din ng mga kaalaman paano gamitin, ano ang Sistema ng edukasyon sa ngayon upang mabigyan rin nila ng angkop na suporta ang bawat mag-aaral. Kailangan rin na magkaroon ng bukas at maayos na komunikasyon ang mga magulan sa paaralan at guro ng mga mag-aaral upang mabisang masolusyonan ang mga suliraning kakaharapin sa pagkatuto.

> Sa mga mananaliksik marapat na ipagpatuloy ang mga nalamang mga saloobin na naranasan ng mag-aaral sa panahon ng makabagong edukasyon, lawakan pa ang sakop ng pag-aaral ng sa gayon ay makapagbigay at makabuo ng isang mas kapakipakinabang at mabisang solusyon upang matugunan ang suliranin. 


\section{Sanggunian}

Agung, A. S. S. N., Surtikanti, M. W., \& Quinones, C. (2020). View of students' perception of online learning during COVID-19 pandemic: A case study on the english students of STKIP Pamane Talino. Journal of Social Sciences and Humanities, 10(2).

Bernard, A., Bowen, W.G., Chingos, M. M., Lack, K. A., \& Nygren, T. I. (2014). Interactive learning online at public universities: Evidence from a six-campus randomized trial. Journal of Policy Analysis \& Management, 33(1), 94-111.

Bilbao, P. L., Ricafort, N. S., \& Calagui, L. S. (2020). The teaching profession. pp. 152 republic act no. 7722 association of higher education institutions region-IVA (AHEIR).

Burke, L. (2020, October 27). Long-term online learning in pandemic may impact students' well-being. Inside Higher Ed. www.insidehighered.com.

De La Cruz, C. (2020, September 12). Online learning in the time of COVID-19: A day in the life of a college student. SPOT.PH.

del Castillo, F. (2021) COVID-19 impact on learners: challenges and responses. Journal of Public Health, 43(3), 539-540.

Dwidienawati, D., Sri, B., Abdinagoro, S., \& Gandasri, D. (2020). E-learning implementation during the COVID-19 outbreak: The perspective of students and lecturers' optimization view project research in entrepreneurship on educational and research institution view project. Journal of the Social Sciences.

Emelinda, A. (2020). Campus press learning in the new normal through the eyes of Filipino students. The Manila Times.

Fitri, B. (2020). Promoting self-directed learning through the implementation of cooperative learning in a higher education blended learning environment. Johannesburg, SA: Doctoral dissertation at North-West University.

Hiltz, S. R., \& Turoff, M. (2005). Education goes digital: The evolution of online learning and the revolution in higher education. Communications of the ACM, 48(10), 59-64.

Jaggars, S. S. (2014). Choosing between online and face-to-face courses: Community college student voices. American Journal of Distance Education, 28(1), 27-38. Retrieved May 14, 2021 from http://ccrc.tc.columbia.edu/media/k2/attachments/online-demand-student-voices.pdf

Khan, T., \& Jumani, D. (2010). Assessing social presence in asynchronous text-based computer conferencing. Journal of Asynchronous Learning Networks, 5(2).

Kneller, L. C. (2009). Constructivism learning theory. Simply Psychology.

Laili, R. N., \& Nashir, M. (2021). Higher education students' perception on online learning during covid-19 pandemic. Jurnal Ilmu Pendidikan, 3(3), 689-697. Retrieved May 15, 2021 from https://journals.lww.com/md-journal/Fulltext/2021/02190/Students_perception_of_online_learning_du ring_the.87.aspx

Leo, D. (2021). PH internet speed moves up in global rankings. The Manila Times.

Mallillin, L. L. D., Mendoza, L. C., Mallillin, J. B., \& Felix (2020). Implementation and readiness of online learning pedagogy: A transition to covid 19 pandemic. European Journal of Open Education and E-Learning Studies, 5(2). Retrieved May 14, 2021 from https://oapub.org/edu/index.php/ejoe/article/view/3321

Siemens, G. (2004). Connectivism: A learning theory for the digital age. E-learning Space.

Subekti, A. S., \& Wacana, U. K. D. (2021). Covid-19-triggered online learning implementation: Pre-service english teachers' beliefs. Metathesis: Journal of English Language, Literature, and Teaching, 4(3), 232. 
Dadacay, A., Guba, A., Costiniano, A., Gumba, K., Lendio, J., \& Lopez, C. D.

86 Consortia Academia Publishing (A partner of Network of Professional Researchers and Educators) 